\title{
Paul BAUER et Mathilde DARLEY (dir.), Frontières de l'Union Européenne : franchissements et résistances
}

\section{Mathieu Petithomme}

\section{(2) OpenEdition}

Édition électronique

URL : https://journals.openedition.org/remi/4313

DOI : $10.4000 /$ remi.4313

ISSN : $1777-5418$

Éditeur

Université de Poitiers

\section{Édition imprimée}

Date de publication : 1 avril 2008

Pagination : 160-162

ISBN : 978-2-917-627-48-2

ISSN : 0765-0752

Référence électronique

Mathieu Petithomme, " Paul BAUER et Mathilde DARLEY (dir.), Frontières de I'Union Européenne: franchissements et résistances ", Revue européenne des migrations internationales [En ligne], vol. 24 $\mathrm{n}^{\circ} 1$ | 2008, mis en ligne le 27 novembre 2008, consulté le 16 avril 2022. URL : http:// journals.openedition.org/remi/4313 ; DOI : https://doi.org/10.4000/remi.4313

Ce document a été généré automatiquement le 16 avril 2022.

(c) Université de Poitiers 


\title{
Paul BAUER et Mathilde DARLEY (dir.), Frontières de l'Union Européenne : franchissements et résistances
}

\author{
Mathieu Petithomme
}

\section{RÉFÉRENCE}

Paul BAUER et Mathilde DARLEY (dir.), Frontières de l'Union Européenne : franchissements et résistances, Prague, Cefres, 2007, 293 p., ISBN : 978-80-86311-00-5

1 L'ouvrage édité par Paul Bauer et Mathilde Darley réunit un ensemble de travaux portant sur la problématique des frontières de l'Union Européenne (UE) dans le contexte de l'élargissement. Dans la préface de l'ouvrage, Hastings Donnan résume bien l'importance d'une analyse plus précise de la question des frontières de l'UE. Il rappelle au lecteur que les frontières ne nous renseignent pas simplement sur les limites d'un espace sociopolitique donné, mais qu'elles contribuent plus fondamentalement à éclairer le « centre » à partir de la "périphérie ». En d'autres termes, « en comprenant ce que les frontières excluent, nous apprenons autant sur ce qu'elles contiennent » (p.15). Percevoir les rapports de forces et les enjeux associés aux zones frontalières éclaire donc indirectement les processus de formation et de redéfinition des limites de l'appartenance nationale, cette « communauté imaginée » selon Benedict Anderson.

2 L'objectif principal de l'entreprise est donc de renouveler le débat sur les frontières méridionales et orientales de l'Union en se centrant sur les processus politiques de coopération transfrontalière, les stratégies locales ainsi que les représentations des acteurs sociaux. Les coordinateurs du projet prétendent donc définir théoriquement et illustrer empiriquement l'efficacité de la méthode d'analyse géographique dans sa capacité à articuler diverses échelles tout en permettant de conceptualiser plus 
pertinemment les processus sociopolitiques à l'œuvre. Suivant la perspective choisie, les coordinateurs préfèrent ainsi la notion d'espace frontière à celle de ligne frontière, plus restrictive. L'espace frontalier compris au sens large est donc choisi comme une porte d'entrée privilégiée du travail collectif, comme « laboratoire scientifique » permettant notamment de spécifier l'impact de l'ouverture ou de la fermeture d'un territoire donné sur la définition des identités locales et nationales.

Deux axes de recherche sont ainsi privilégiés: d'une part, la question du franchissement des frontières et de l'adaptation des acteurs sociaux aux modifications des conditions de passage, et d'autre part, les représentations sociales et les dynamiques de production/délimitation des identités associées aux frontières. L'espace frontière est ainsi traité tant au sens littéral et pratique du terme qu'au sens figuré et symbolique. L'ouvrage collectif est divisé en trois parties complémentaires. Dans un premier temps, les représentations sociales de la frontière et les processus identitaires qui lui sont associés sont étudiés au travers de perspectives historiques. Dans un second temps, la focale est déplacée sur les acteurs sociaux et les pratiques de dérogation aux règles de franchissement. Finalement, la troisième partie s'intéresse plus spécifiquement aux échanges transfrontaliers et aux politiques de voisinage de l'UE. Étant donné qu'il est impossible de rendre compte de manière compréhensive des tenants et des aboutissants de chacun des dix chapitres du volume, nous centrerons notre propos sur les éléments les plus intéressants à nos yeux.

4 Le premier chapitre rédigé par Jocelyn Parot s'intéresse au cas de la Finlande en soulignant comment la fin de la Guerre Froide et le processus d'intégration européenne ont participé conjointement à la redéfinition collective de la place de la nation finlandaise au sein de son environnement géographique. Autrefois perçue subjectivement comme "quelque part» entre l'Ouest et l'Est, la Finlande s'est progressivement convertie en espace frontière de l'UE. Grâce à une utilisation raisonnée de la littérature finlandaise et internationale sur la frontière séparant la Finlande de la Russie, et au travers d'une étude de cas portant sur les principaux sites frontaliers, Jocelyn Parot met l'emphase sur la mémoire collective attachée à l'espace frontalier, notamment au travers de la résistance à l'influence communiste. Alors que l'espace frontalier se définissait jusqu'alors en référence au passé, sa signification doit aujourd'hui être reconstruite suivant les enjeux contemporains et la situation périphérique de la Finlande au sein d'une Europe élargie.

5 Dans un deuxième chapitre, Eduard Rodriguez- Martin analyse le cas des enclaves espagnoles de Ceuta et Melilla afin de comprendre la spécificité sociopolitique de territoires frontaliers européens paradoxalement situés sur le continent africain. L'auteur expose comment l'isolement de ces enclaves et la tendance à un renforcement de l'autarcie et des barrières tant physiques que symboliques qui les séparent du Maroc, contrastent cependant en pratique avec la dépendance alliant les habitants des deux villes avec les populations autochtones.

6 Eduard Rodriguez-Martin démontre comment les Espagnols ont promu de manière apparemment contradictoires des mesures permissives (accès journalier à l'enclave des travailleurs marocains, tolérance à l'égard des trafics transfrontaliers...), tout autant que des politiques répressives (renforcement des contrôles, construction d'un mur de séparation...). Le point central de son argumentation est lié au fait que la frontière principale entre Espagnols et Marocains n'est pas simplement physique, mais plutôt juridique et sociale, dans le sens où bien que de nombreux marocains participent 
activement à la vie des deux enclaves, ils pâtissent d'une différenciation et d'une ségrégation interne résultant de l'accès inégal à la nationalité et à la citoyenneté. Paradoxalement, en promouvant la « reconnaissance de la différence » des Marocains, la politique multiculturelle a joué un rôle privilégié pour les administrations locales afin de résister à l'assimilation et de marginaliser socialement les autochtones.

Cette relation dialectique entre frontières physiques externes et frontières symboliques internes se retrouve dans le texte de Siarhei Liubimau qui porte sur les expériences vécues des exilés biélorusses en Pologne. Utilisant une approche anthropologique et s'appuyant sur plusieurs entretiens, Liubimau montre comment la minorité biélorusse à Varsovie tend à importer une vision symbolique négative de la frontière séparant leur pays d'origine de la Pologne. Plus qu'une barrière matérielle, au demeurant assez facilement franchissable, c'est une différenciation vécue et imaginée, une charge émotionnelle importante qui est le plus souvent associée à l'espace frontière, entre une Pologne permissive et intégrée à l'UE d'une part, et une Biélorussie répressive et marginalisée internationalement d'autre part.

8 La deuxième partie de l'ouvrage rassemble des travaux s'intéressant plus spécifiquement aux fonctionnements des économies politiques transfrontalières et à leur mutation depuis le dernier élargissement. Bénédicte Michalon caractérise ainsi les nouvelles formes de mobilité qui émergent à la frontière roumano-moldave et l'évolution des stratégies des trafiquants et des commerçants locaux en réponse à une évolution de leur environnement géographique. Son analyse tend à défendre l'idée que les espaces frontières sont beaucoup plus opaques qu'il n'y paraît au premier abord. Alors que la mobilité des Moldaves vers la Roumanie était quasiment impossible durant la Guerre Froide, celle-ci s'est rapidement développée depuis le début des années 1990. Selon l'auteur, l'intégration européenne n'affaiblit pas, mais au contraire, peut renforcer les frontières externes de l'Union. En contribuant à différencier le statut de l'intégré (la Roumanie) de celui de l'exclu (la Moldavie), elle crée indirectement une asymétrie qui, dans le cas étudié, force constamment les Moldaves à s'adapter à l'évolution des conditions d'ouverture et de fermeture de l'espace frontalier. L'analyse de Pauline Carnet portant sur la zone frontalière andalouse en Espagne semble coïncider en de nombreux points avec celle de Bénédicte Michalon. En effet, Pauline Carnet défend l'idée qu'il existe une spécificité socio-économique des espaces frontaliers dans le sens où l'existence de limites politiques et géographiques clairement identifiées favorise le développement de nouvelles opportunités économiques tout en rendant manifeste des disparités sociales profondes auparavant occultées. Les frontières peuvent ainsi bénéficier à certains groupes sociaux tout en portant préjudice à d'autres.

9 La troisième partie de l'ouvrage présente des études de cas portant sur l'application locale de la politique de voisinage de l'UE. Eunice Blavascunas s'intéresse à la frontière de la Biélorussie en proposant une analyse complémentaire de celle de Siarhei Liubimau. Jean Gardin se focalise sur l'espace frontalier entre l'Albanie, la Macédoine et la Grèce, définissant les contradictions de la politique d'intégration de l'UE qui en pratique, doit faire face aux réticences des acteurs locaux, à des stratégies différenciées et à une planification trop souvent inappropriée et loin de la réalité vécue par les populations. En conclusion, Paul Bauer et Mathilde Darley reviennent sur l'importance d'un débat multidimensionnel sur le «fait frontière» qui allie une multiplicité d'approches en les appliquant à différents terrains d'enquêtes. D'une manière générale, 
les études rassemblées au sein de cet ouvrage sont instructives. Le lecteur appréciera la diversité des analyses et l'intérêt de comparaisons entre les frontières africaines, balkaniques et slaves de l'Europe d'aujourd'hui. Comme le rappellent les coordinateurs du volume, les textes réunis sont majoritairement issus de recherches en cours et ne prétendent donc pas clore un débat. Si tel est l'objectif principal du livre celui-ci est plutôt réussi.

\section{AUTEURS}

\section{MATHIEU PETITHOMME}

Institut Universitaire Européen de Florence 